\title{
PKM: A Pairwise Key Management Scheme for Wireless Sensor Networks ${ }^{\star}$
}

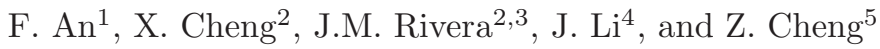 \\ 1 Institute of Computing Technology, Chinese Academy of Sciences, \\ P.O. Box 2704, Beijing 100080, China \\ anfengguang@sof tware.ict.ac.cn \\ 2 Department of Computer Science, The George Washington University, \\ 801 22nd St. NW, Washington, DC 20052, USA \\ cheng@gwu . edu \\ 3 United States Army, Washington, DC, USA \\ jose.rivera@us.army.mil \\ 4 Department of Systems and Computer Science, \\ Howard University, Washington, DC, USA \\ lij@scs.howard.edu \\ 5 National Taxation Bureau of Rizhao City, \\ Shandong Province 276826, China \\ green_sd@163.com
}

\begin{abstract}
Sensor networks are characterized by strict resource limitations and large scalability. Many sensor network applications require secure communication, a crucial component, especially in harsh environments. Symmetric key cryptography is very attractive in sensor networks due to its efficiency, but establishing a shared key for communicating parties is very challenging. The low computational capability and small storage budget within sensors render many popular public-key based key distribution and management mechanisms impractical. In this paper, we propose and analyze a truly in-situ key management scheme for large scale sensor networks, called: Public Key Management (PKM). In this scheme, we deploy service and worker sensors. The service sensors contain a key space, while worker sensors are deployed blind, with no predeployment knowledge. Worker sensors obtain security information from service sensors through a secure channel after deployment. After obtaining security information, worker sensors compute shared keys with their neighbors. For security reasons, service sensors erase stored key space information after deployment. During this procedure, PKM shifts a large amount of computational overhead from worker sensors to service sensors, thus conserving worker sensors' resources. PKM's performance, in terms of storage, computational overhead and resiliency, is very good.
\end{abstract}

Keywords: Sensor networks, security, key management, key distribution.

* The research of Dr. Xiuzhen Cheng is supported by NSF CAREER Award No. CNS-0347674.

X. Lu and W. Zhao (Eds.): ICCNMC 2005, LNCS 3619, pp. $992-10012005$.

(C) Springer-Verlag Berlin Heidelberg 2005 


\section{Introduction}

Today's smart sensors are plagued by strict resource limitations (battery, memory, CPU, etc.). For example, the MICA2 Berkeley mote has an 8-bit, 7.3828MHz Atmega 128L processor with $4 \mathrm{~KB}$ SRAM and 128KB ROM 11. However, these sensors are usually deployed in large scale with high density for monitoring and control. These endemic characteristics create challenging problems in establishing secure communication in sensor networks.

Many sensor network applications require security assurance. Due to its efficiency, symmetric key cryptography is very attractive in sensor networks. However, establishing shared keys between neighboring sensors cannot be considered trivial. The well-established Public Key Infrastructure (PKI) key distribution and management schemes are not applicable due to high computational overhead and large memory requirement. As reported by Carman et. al 4, a middleranged processor such as the Motorola MC68328 "DragonBall" consumes 42mJ $(840 \mathrm{~mJ})$ for RSA encryption (digital signature) and $0.104 \mathrm{~mJ}$ for AES, when the key size for both cases is 1024 bits.

A few key pre-distribution protocols for shared key construction have been proposed in literature 6 67/9] but they may not scale well to large sensor networks or may require strict deployment knowledge for better scalability. Further, all these schemes require some kind of security information to be pre-loaded to the memory of a sensor, assuring storage space waste, since some of the information may never be used during the lifetime of the sensor. In this paper we explore in-situ key computation, instead of security information pre-distribution. To achieve this goal we are willing to sacrifice the "Key Space Carriers", the service sensors. In the initial phase (pre-deployment), worker sensors do not need security information. They actualize their security posture after deployment by interacting with the service sensors. A secure channel is established for secure information transfer between a service sensor and a worker sensor. Once this interaction is completed, service sensors will erase the stored key space. This procedure exploits the asymmetric feature in the computational overhead of Rabin's crypto-system, thus shifting most of the computational burden to the service sensors.

This paper is organized as follows: we briefly overview related works in Section 2, our PKM scheme is proposed in Section 3, public key assisted secure channel establishment protocol is presented in Section 4, we analyze our protocol in Section 5 and conclude our paper in Section 6 .

\section{Related Work}

In this section, we briefly survey the following shared key establishment schemes: random key pre-distribution, symmetric matrix based (Blom's method based) key computation and deployment knowledge based key management.

The pioneer work on random key pre-distribution for sensor networks is proposed by Eschenauer and Gligor in 9. A large key pool $K$ is computed offline 
and each sensor picks $k$ keys randomly from $K$ without replacement before deployment. These $k$ keys form the key ring of the sensor. After deployment, a sensor establishes a shared key with a neighbor if their key rings have at least one key in common. The security of random key pre-distribution is enforced by [5] in which $q>1$ common keys are required to establish a shared key. These $q$ keys are hashed into one key to achieve better resiliency to sensor capture.

Du et. al [6] is the first to apply Blom's scheme for shared key establishment in sensor networks. Blom's scheme is based on the computation of a symmetric matrix which provides a key space for all sensors that possess a public and a private share of the key space. In [6], $\omega$ key spaces instead of one key space is precomputed and each sensor stores the private/public shares of $\tau$ key spaces. These $\tau$ key spaces are randomly selected from the $\omega$ key spaces without replacement. If two sensors share information from one common key space, they can establish a shared key after exchanging their public shares. This scheme combines the idea of random key pre-distribution in 9] with Blom's method. We will elaborate Blom's scheme in the next section.

To improve scalability, a deployment knowledge based key management approach is proposed in [7]. In this scheme, multiple deployment points are identified in the sensor network and for each deployment point, a key space is precomputed. Neighboring deployment points have a number of keys in common. In other words, their key spaces consist of common keys. All sensors are grouped before deployment and each group corresponds to one deployment point. Each sensor randomly picks $k$ keys from the key space of its group. After deployment, sensors in close neighborhood have a high probability of sharing a common key. This scheme places strong requirements on deployment, but achieves better scalability compared with those proposed in [6] .

A geographic information based key management protocol is designed in [10. A general framework for establishing pairwise keys in sensor networks is studied in [12], which is based on the polynomial-based key pre-distribution protocol proposed by [3]. A location-aware deployment model for shared key establishment is presented in 11 .

Our work is different from all those mentioned above in that it is truly an in-situ key management scheme for sensor networks. We do not require any keyrelated information to be pre-distributed to worker sensors. Instead, we randomly deploy service sensors that convey security information to the worker sensors in the neighborhood. This protocol has better scalability, with no requirement of deployment knowledge.

\section{PKM: The Pairwise Key Management Scheme}

In this section, we propose PKM, a key management protocol for establishing pairwise keys between neighboring sensors. This protocol is based on Blom's $\lambda$ secure key management scheme [2, which has been well-tailored for light-weight sensor networks by [6]. In the following, we will give an overview on Blom's scheme based on [6]. 


\subsection{Blom's Key Management Scheme}

Let $G$ be a $(\lambda+1) \times M$ matrix over a finite field $G F(q)$, where $q$ is a large prime. The connotation of $M$ will become clear later. $G$ is public, with each column called a public share. Let $D$ be any random $(\lambda+1) \times(\lambda+1)$ symmetric matrix. $D$ must be kept private, which is known to the network service provider only. The transpose of $D \cdot G$ is denoted by $A$. That is, $A=(D \cdot G)^{T} \cdot A$ is private too, with each row called a private share. Since $D$ is symmetric, $A \cdot G$ is symmetric too. If we let $K=\left(k_{i j}\right)=A \cdot G$, we have $k_{i j}=k_{j i}$, where $k_{i j}$ is the element at the $i$ th row and the $j$ th column of matrix $K, i, j=1,2, \cdots, M$.

The basic idea of Blom's scheme is to use $k_{i j}$ as the secret key shared by sensor $i$ and sensor $j$. $D$ and $G$ jointly define a key space $(D, G)$. Any public share in $G$ has a unique private share in $A$, which form a so-called crypto pair. For example, the $i$ th column of a $G$, and the $i$ th row of $A$ form a crypto pair and the unique private share of the $i$ th column of $G$, a public share, is the $i$ th row of $A$. Two sensors whose crypto pairs are obtained from the same key space can compute a shared key after exchanging their public shares. From this analysis, it is clear that $M$ is the number of sensors that can compute their pairwise keys based on the same key space.

In summary, Blom's scheme states the following protocol for sensors $i$ and $j$ to compute $k_{i j}$ and $k_{j i}$, based on the same key space:

- Each sensor stores a unique crypto pair. Without loss of generality, we assume sensor $i$ gets the $i$ th column of $G$ and the $i$ th row of $A$, denoted by $g_{k i}$ and $a_{i k}$, where $k=1,2, \cdots, \lambda+1$, respectively. Similarly, sensor $j$ gets the $j$ th column of $G$ and the $j$ th row of $A$, denoted by $g_{k j}$ and $a_{j k}$, where $k=1,2, \cdots, \lambda+1$, respectively.

- Sensor $i$ and sensor $j$ exchange their stored public share drawn from their crypto pairs as plain texts.

- Sensor $i$ computes $k_{i j}$ as follows:

$$
k_{i j}=\sum_{k=1}^{\lambda+1} a_{i k} \cdot g_{k j} ;
$$

Similarly, sensor $j$ computes $k_{j i}$ by

$$
k_{j i}=\sum_{k=1}^{\lambda+1} a_{j k} \cdot g_{k i} .
$$

Blom's key management scheme ensures the so-called $\lambda$-secure property, which means that the network should be perfectly secure as long as no more than $\lambda$ sensors are compromised. This requires that any $\lambda+1$ columns of $G$ must be linearly independent. A good candidate of $G$ can be the Vandermonde matrix:

$$
G=\left[\begin{array}{ccccc}
1 & 1 & 1 & \cdots & 1 \\
s & s^{2} & s^{3} & \cdots & s^{M} \\
s^{2} & \left(s^{2}\right)^{2} & \left(s^{3}\right)^{2} & \cdots & \left(s^{M}\right)^{2} \\
& \vdots & \\
s^{\lambda} & \left(s^{2}\right)^{\lambda} & \left(s^{3}\right)^{\lambda} & \cdots & \left(s^{M}\right)^{\lambda}
\end{array}\right],
$$


where $s$ is the primitive element of $G F(q)$ for some large prime number $q$ with $M<q$. Note that only the second element of each column needs to be disseminated as a public share, from which other elements can be easily restored.

\subsection{PKM: The Pairwise Key Management Protocol}

Let each service sensor $I$ carry a key space $(D, G)_{I}$ before deployment. Service sensors can announce their existence through beacon broadcasting after deployment, or we can allow worker sensors to query their neighborhood (can be multi hop) for service sensors. The topic on how to identify service sensors in the neighborhood is beyond the scope of this paper. We assume that there exists an idealized protocol, such that each worker sensor can be connected through single or multi hop to at least one service sensor.

In order for a worker sensor to obtain a column of $G$ and the corresponding row of $A$ from a service sensor, we need a secure channel between the worker sensor and the service sensor. A public-key encryption based protocol for secure channel establishment will be proposed in the next section. For now, we assume there exists a shared key $K_{s}$ between a worker sensor and its service sensor.

Our pairwise key establishment protocol can be stated as follows.

- Each worker sensor sends a request to the service sensor, asking for a crypto pair containing a public and a private share. This message can be secured by $K_{s}$.

- Upon receiving a request from sensor $i$, service sensor $I$ selects an unused crypto pair and then transmits it to $i$. This message must be encrypted by $K_{s}$.

- Two neighboring sensors exchange their public shares obtained from the same key space (through the same service sensor) to compute their pairwise key based on Blom's scheme.

This procedure can be further secured with the introduction of nonces to avoid replay attacks.

Note that in this protocol we do not ensure a globally unique id for each service sensor. But we do require that service sensors connected to a common worker sensor be uniquely identified in order for the sensor to tell the key spaces apart. Further, the $G$ s for different service sensors can be the same, as long as the key spaces associated with a single worker sensor can be clearly identified; because of this, this protocol scales well to very large sensor networks.

\section{Public Key Assisted Secure Channel Establishment}

It is clear that the applicability of our PKM protocol depends on the availability of a secure channel between a worker sensor and the corresponding service sensor since the worker sensor needs its private share for pairwise key computation. In this section, we propose a public key assisted key exchange protocol to establish a secret key $K_{s}$ between a worker sensor and a service sensor. 
Our simple idea is based on the following observations. First, pairwise key establishment is a bootstrapping protocol that ensures a newly deployed sensor network to initiate a secure infrastructure. Thus, we can simply assume all worker sensors and service sensors are trust-worthy if they are deployed together. Rekeying and key establishment for future deployment will be studied in future research. Second, there is no a priori security information between a worker sensor and a service sensor. For the sensor network under consideration, public-key based key exchange for secret key establishment is the only choice for us. Third, since worker sensors are supposed to operate for an un-deterministically long time while service sensors can die after deployment, cryptographic algorithms that shift large amount of the computational overhead to the service sensors are preferred.

Our public-key assisted secret key exchange is based on the Rabin cryptosystem [14. Rabin's scheme has asymmetric computational cost. Its encryption operation is several hundred times faster than RSA, but it's decryption time is comparable to that of RSA. The security of Rabin's scheme is based on the factorization of large numbers, thus, it is also comparable to that of RSA.

\subsection{Rabin's Scheme}

Rabin's scheme is an asymmetric cryptosystem where we need to compute both a public and a private key.

Key Generation: Choose two large distinct primes $p$ and $q$ such that $p \equiv q \equiv 3$ $\bmod 4 .(p, q)$ is the private key while $n=p \cdot q$ is the public key.

Encryption: For the encryption, only the public key $n$ is needed. Let $p$ be the plain text that is represented as an integer in $Z_{n}$. Then the cipher text $c=p^{2}$ $\bmod n$.

Decryption: Since $p \equiv q \equiv 3 \bmod 4$, we have

$$
m_{p}=c^{\frac{p+1}{4}} \bmod p
$$

and

$$
m_{q}=c^{\frac{q+1}{4}} \bmod q .
$$

By applying the extended Euclidean algorithm, $y_{p}$ and $y_{q}$ can be computed such that $y_{p} \cdot p+y_{q} \cdot q=1$.

From the Chinese Remainder Theorem, four square roots $+r,-r,+s,-s$ can be obtained:

$$
\begin{aligned}
r & =\left(y_{p} \cdot p \cdot m_{q}+y_{q} \cdot q \cdot m_{p}\right) \bmod n \\
-r & =n-r \\
s & =\left(y_{p} \cdot p \cdot m_{q}-y_{q} \cdot q \cdot m_{p}\right) \bmod n \\
-s & =n-s
\end{aligned}
$$


Note that encryption in Rabin's scheme requires only one squaring, which takes less time and energy compared to RSA, which requires multiple squarings and multiplications. Also note that decryption in Rabin's scheme produces three false results in addition to the correct plain text. This can be easily overcome in practice by adding pre specified redundancy to the plain text before encryption.

Furthermore, a careful reader may wonder why we did not choose RSA with a small prime number such as 3 as the public encryption exponent. RSA is preferable since it has gone through extensive cryptanalysis. But unfortunately a small encryption exponent is not secure when the same message needs to be sent out to multiple destinations or the plain text is too short [13]. This may be the case in our application scenario since a request may be short and it may be delivered to multiple service nodes.

\subsection{SKE: Secret Key Exchange Protocol}

Based on Rabin's scheme described in Subsection 4.1, we propose the following secret key exchange protocol between worker sensors and service sensors.

- For each service sensor, computes two large distinct primes $p$ and $q$ such that $p \equiv q \equiv 3 \bmod 4 . n=p \cdot q$ is the public key and $(p, q)$ is the private key. Broadcasts $n$ to all associated worker sensors. Note that $p$ and $q$ can be computed off-line by a supercomputer for all service nodes.

- For each associated service sensor $I$, a worker sensor picks $K_{s}$, computes $E_{n}\left(K_{s} \| R\right)=K_{s}^{2} \bmod n$, where $R$ is a predefined bit pattern to resolve the ambiguity in Rabin's decryption, and transmits $E_{n}\left(K_{s}\right)$ to $I . K_{s}$ is the shared key between the worker sensor and service sensor $I$.

- Upon receiving the $E_{n}\left(K_{s}\right)$ from a worker sensor, the service sensor computes $D_{(p, q)}\left(E_{n}\left(K_{s}\right)\right)$ based on Rabin's decryption algorithm.

Note that this protocol only requires executing the encryption algorithm once by the worker sensor and no decryption is involved. Furthermore, encryption in Rabin's scheme is extremely simple. This can save energy to extend the operational time of worker sensors.

\section{Analysis}

In this section, we briefly analyze PKM along the lines of connectivity, overhead and security. This study is motivated by 6 .

\subsection{Connectivity Analysis}

The graph formed by all secure links is called a key-sharing graph, denoted by $G$. For a network to function properly, $G$ must be connected. If a sensor establishes shared keys with all neighbors, then the induced graph $G$ is connected if the original topology is connected. 
In reality it may not be efficient to require all links to be active all the time, mainly due to the contention and delay caused by the MAC layer. This is especially true in a dense sensor network where each sensor has tens of immediate neighbors. Usually it suffices if a subset of links are secured and all secure links form a connected graph. In other words, a worker sensor establishes secure links with a subset of neighbors and communicates with other neighbors through multi hop transmission. But what is the expected degree of each worker sensor in the key-sharing graph $G$ such that $G$ is connected? Or connected with high probability? Erdős and Rényi's random graph theory can help us to answer this question.

Let $d$ be the expected degree for a worker sensor in $G$. The connectivity theory of Erdős and Rényi [8] states that $G$ is connected with a probability $P_{c}$ for a network with $N$ nodes when $N$ is large if

$$
d=\frac{N-1}{N}[\ln (N)-\ln (-\ln (P c))] .
$$

Actually $d$ should be sufficiently large such that $G$ is connected with high probability. This analysis can provide a guideline when applying our PKM key management protocol to a newly deployed sensor network.

\subsection{Overhead Analysis}

In this Subsection, we will study the memory usage, and the communication and computation overheads of a worker sensor in our PKM scheme.

The storage budget allocated for shared key establishment in a sensor node impacts the security level of our PKM scheme. Blom's symmetric key computation algorithm is $\lambda$-secure. Thus, the larger the $\lambda$, the better the security. However, the total memory budget and the number of crypto pairs a worker sensor must store for ensuring global connectivity places constraints on the size of $\lambda$.

A worker sensor needs to locate service sensors in the neighborhood, establish secure channels with them and then obtain crypto pairs for shared key computation. Service sensors will broadcast their existence, this way worker sensors do not need to query their neighborhood. It is clear that all these communications are confined locally.

Computing a shared key requires $2 \lambda$ modular multiplications, with $\lambda$ of them for restoring all the elements in the public share and the other $\lambda$ of them for computing the shared key. A worker sensor also needs $\lambda$ modular addition for a shared key computation.

\subsection{Security Analysis}

In this subsection, we study the resiliency of PKM to sensor capture through probability analysis. We assume that if a sensor is captured, all pairwise keys shared by this sensor and others will be compromised. Further, we assume all 


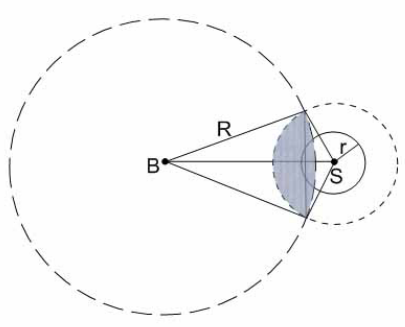

Fig. 1. The overlapping area is colored gray. In this example, $t=2 . B$ is the center of the disk all $x$ compromised nodes reside. $S$ is the service sensor whose key space information may be released by compromised nodes

sensors are distributed randomly and uniformly in a two-dimensional area A. Note that we only consider the simple scenario when a service sensor provides security information to all worker sensors within $t$ hops away and a worker sensor asks for a crypto pair from each service sensor within $t$ hops away. Let $r$ be the transmission range.

Assume that $x$ number of nodes have been captured. Since Blom's scheme is $\lambda$-secure, we assume $x>\lambda$. There exists two scenarios we need to consider:

- Case A: The $x$ compromised nodes are independently and randomly distributed in the whole area $\mathrm{A}$;

- Case B: The $x$ compromised nodes are independently and randomly distributed in a small area B.

We denote the areas of $\mathrm{A}$ and $\mathrm{B}$ by $A$ and $B$, respectively. Let $P$ be the probability that any key space is compromised. In other words, $P$ is the probability that more than $\lambda$ number of compromised sensors have obtained information from a service sensor $S$ carrying the key space. Let $p$ be the probability that each compromised node carries information about $S$. We have:

$$
P=\sum_{j=\lambda+1}^{x}\left(\begin{array}{l}
x \\
j
\end{array}\right) p^{j}(1-p)^{x-j} .
$$

Next, we will study $p$ for each case:

Case A: Since $S$ provides information to all worker sensors within $t$ hops away, it is possible that a sensor within the disk area of radius $t r$ centered at $S$ contains information about $S$. Thus $p \leq \frac{\pi(t r)^{2}}{A}$.

Case B: For simplicity, we assume area $B$ is a circle with radius $R>2 t r$ centered at location $B$. Let $y$ be the Euclidean distance between $S$ and $B$. When $y>R+t r$, no information on $S$ is released by the $x$ captured nodes. Therefore $p=0$. Otherwise, $p \leq \frac{\theta}{B}$, where $\theta$ is the overlapping area, as shown by the gray area in Fig. 1. 


\section{Conclusion}

In this paper, we have proposed and analyzed "PKM", an in-situ key management protocol for sensor networks. PKM is based on the idea of sacrificing a number of service sensors so a large amount of computational and storage overhead can be shifted away from worker sensors. Since worker sensors require no information to be pre-loaded, PKM scales well to large sensor networks. As a future research, we will analyze the performance of PKM with more general assumptions.

\section{References}

1. http://www.xbow.com/Products/Product_pdf_files/Wireless_pdf/6020-0042-06_B _MICA2.pdf.

2. R. Blom, An optimal class of symmetric key generation systems, Advances in Cryptology: Proceedings of EUROCRYPT 84 (Thomas Beth, Norbert Cot, and Ingemar Ingemarsson, eds.), Lecture Notes in Computer Science, Springer-Verlag, vol. 208, pp.335-338, 1985.

3. C. Blundo, A. De Santis, A. Herzberg, S. Kutten, U. Vaccaro, and M. Yung, Prefectly-Secure Key Distribution for Dynamic Conferences, Advances in Cryptoglogy - CRYPTO'92, LNCS 740, pp. 471-486, 1993.

4. D. W. Carman, P. S. Kruus, and B. J. Matt, Constraints and Approaches for Distributed Sensor Network Security, NAI Labs Technical Report No. 00-010, September, 2000.

5. H. Chan, A. Perrig, and D. Song, Random Key Predistribution Schemes for Sensor Networks, IEEE SP 2003.

6. W. Du, J. Deng, Y.S. Han, and P.K. Varshney, A pairwise key pre-distribution scheme for wireless sensor networks, CCS'03, pp. 42-51, October 27-31, 2003, Washington DC, USA.

7. W. Du, J. Deng, Y.S. Han, S. Chen, and P.K. Varshney, A Key Management Scheme for Wireless Sensor Networks Using Deployment Knowledge, IEEE INFOCOM 2004.

8. Erdős and Rényi, On Random Graphs I, Publ. Math. Debrecen, 6:290-297, 1959.

9. L. Eschenauer and V.D. Gligor, A Key-Management Scheme for Distributed Sensor Networks, CCS'02, pp.41-47, November 18-22, 2002, Washington DC, USA.

10. S. C.-H Huang, M.X. Cheng, and D.-Z. Du, GeoSENS: Geo-based SEnsor Network Secure Communication Protocol, manuscript, 2004.

11. D. Liu and P. Ning, Location-Based Pairwise Key Establishments for Static Sensor Networks, Proc. 1st ACM Workshop on Security of Ad Hoc and Sensor Networks, pp. $72-82,2003$.

12. D. Liu and P. Ning, Establishing Pairwise Keys in Distributed Sensor Networks, ACM CCS'03, pp. 52-60, 2003.

13. A. J. Menezes, P. C. van Oorschot, and S. A. Vanstone, Handbook of Applied Cryptography, CRC Press, 1997.

14. M.O. Rabin, Digitalized signatures and public key functions as intractable as factorization, MIT/LCS/TR-212, MIT, 1979. 